\title{
PURSUIT CURVES AND ORDINARY DIFFERENTIAL EQUATIONS
}

This paper deals with the differential equations which describe curves of pursuit, in which the pursuer's velocity vector always points directly towards the pursued. We use the Laplace Transform method to solve the classic problem of four mice pursuit.

Key words: pursuit curve, differential equation, the Laplace Transform method

\section{Introduction}

A curve of pursuit is the path an object takes when chasing another object. The problem of pursuit probably originated with Leonardo da Vinci. He was the first one to study this problem when the pursued moved along a straight line. The general case was studied in 1732 by Pierre Bouguer (1698-1758), the French scientist. The problem deals with a pirate ship pursuing a merchant vessel. It is to find the curve by which a pirate ship moves while pursuing a merchant vessel, supposing that the speeds of the two vessels are always in the same ratio. Let us formulate this problem mathematically.

\section{Definition}

Let $\alpha$ and $\beta$ be plane curves parametrized on an interval $a<t<$ $b$. We say that $\alpha$ is a pursuit curve of $\beta$ provided that

(i) the velocity vector $\alpha^{\prime}(t)$ points towards the point $\beta(t)$ for $a<t<$ $<b$, that is $\alpha^{\prime}(t)$ is a multiple of $\alpha(t)-\beta(t)$,

(ii) the speeds of $\alpha$ and $\beta$ are related by $\left\|\alpha^{\prime}\right\|=k\left\|\beta^{\prime}\right\|$, where $k$ is a positive constant. We call $k$ the speed ratio.

$A$ capture point is a point p for which $\boldsymbol{p}=\alpha\left(t_{1}\right)=\beta\left(t_{1}\right)$ for some $t_{1}$.

When the speed ratio $k$ is larger than 1 , the pursuer travels faster than the pursued. Although this would usually be the case in a physical situation, it is not a necessary assumption for the mathematical analysis of the problem.

We derive differential equations for pursuit curves in terms of coordinates.

Lemma. Write $\alpha=(x, y)$ and $\beta=(f, g)$, and assume that $\alpha$ is a pursuit curve of $\beta$. Then

$$
\left(x^{\prime}\right)^{2}+\left(y^{\prime}\right)^{2}=k^{2}\left[\left(f^{\prime}\right)^{2}+\left(g^{\prime}\right)^{2}\right]
$$

and

$$
x^{\prime}(y-g)-y^{\prime}(x-f)=0 .
$$

Proof. Equation (1.1) is the same as $\left\|\alpha^{\prime}\right\|=k\left\|\beta^{\prime}\right\|$. To prove (1.2), we observe that $\alpha(t)-\beta(t)=(x(t)-f(t), y(t)-g(t))$ and $a^{\prime}(t)=\left(x^{\prime}(t), y^{\prime}(t)\right)$. Note that the vector $(-y(t)+g(t), x(t)-$ $f(t))$ is perpendicular to $\alpha(t)-\beta(t)$. The condition that $\alpha^{\prime}(t)$ is a multiple of $\alpha(t)-\beta(t)$ is conveniently expressed by saying that $\alpha^{\prime}(t)$ is perpendicular to $(-y(t)+g(t), x(t)-f(t))$, that is,

$$
\begin{aligned}
0 & =\left(x^{\prime}(t), y^{\prime}(t)\right) \cdot(-y(t)+g(t), x(t)-f(t))= \\
& =x^{\prime}(t)(-y(t)+g(t))+y^{\prime}(t)(x(t)-f(t)) .
\end{aligned}
$$

Then (1.3) is equivalent to (1.2).

Next, we specialize to the case when the curve of the pursued is a straight line.

\section{Example 1}

Assume that the curve $\beta$ of the pursued is a vertical straight line passing through the point $(a, 0)$, and that the speed ratio $k$ is larger than 1 . Find the curve a of the pursuer, assuming the initial conditions $\alpha(0)=(0,0)$ and $\alpha^{\prime}(0)=(1,0)$.

Solution. We can parameterize $\beta$ as

$\beta(\mathrm{t})=(a, \mathrm{~g}(\mathrm{t}))$.

Furthermore, the curve $\alpha$ of the pursuer can be parametrized $\alpha(\mathrm{t})=(t, y(t))$

\footnotetext{
* Zuzana Malacka

Department of Mathematics, Faculty of Humanities, University of Zilina, Slovakia, E-mail: zuzana.malacka@fpv.uniza.sk
} 
The condition (1.1) becomes

$$
1+\left(y^{\prime}\right)^{2}=k^{2}\left(g^{\prime}\right)^{2}
$$

and (1.2) reduces to

$$
(y-g)-y^{\prime}(t-a)=0 .
$$

Differentiation of (1.5) with respect to t yields

$$
-y^{\prime \prime}(t-a)=g^{\prime}
$$

From (1.4) and (1.6) we get

$$
1+\left(y^{\prime}\right)^{2}=k^{2}(a-t)^{2}\left(y^{\prime \prime}\right)^{2}
$$

Let $p=y^{\prime}$. Then (1.7) can be rewritten as

$$
\frac{k d p}{\sqrt{1+p^{2}}}=\frac{d t}{|a-t|} \text {. }
$$

This separable first-order equation has the solution

$$
\arg \sinh (p(t))=\frac{1}{k} \ln \left|\frac{a-t}{a}\right|,
$$

for $p(0)=y^{\prime}(0)=0, a \neq 0, t \neq a$, where $t \in(a, \infty)$ for $a<0$, and $t \in(-\infty, a)$

for $a>0$

$$
\begin{aligned}
& \sinh (\arg \sinh (p(t)))=p(t)=y^{\prime}(t)= \\
& \sinh \left(\ln \left|\frac{a-t}{a}\right|^{-\frac{1}{k}}\right)=\frac{1}{2}\left(\left|\frac{a-t}{a}\right|^{-\frac{1}{k}}-\left|\frac{a-t}{a}\right|^{-\frac{1}{k}}\right)
\end{aligned}
$$

For $a<0$ is $a-t<0$, for $a>0$ is $a-t>0$, and

$$
\begin{aligned}
& y^{\prime}(t)=\frac{1}{2}\left(\left|\frac{a-t}{a}\right|^{-\frac{1}{k}}-\left|\frac{a-t}{a}\right|^{-\frac{1}{k}}\right), \\
& y(t)=\frac{a k}{k^{2}-1}+\frac{1}{2}\left(\frac{a k}{k+1}\left(\frac{a-t}{a}\right)^{1+\frac{1}{k}}-\right. \\
& \left.-\frac{a k}{k-1}\left(\frac{a-t}{a}\right)^{1+\frac{1}{k}}\right), \text { for } y(0)=0
\end{aligned}
$$

The curve of the pursuer is then $\alpha(t)=(t, y(t))$, where $y$ is given by (1.11). Since $a\left(t_{1}\right)=\beta\left(t_{1}\right)$ if and only if $t_{1}=a$, the capture point is

$$
p=\left(a, \frac{a k}{k^{2}-1}\right) \text {. }
$$

The graph below depicts the case when $a=1$ and $k$ has the values $1.5,2.0,2.5,3.0$, and 3.5 . As the speed ratio $k$ becomes smaller and smaller, the capture point goes higher and higher [1].

Now we use the Laplace Transform method for solving to the classic four mice pursuit problem [2-4].

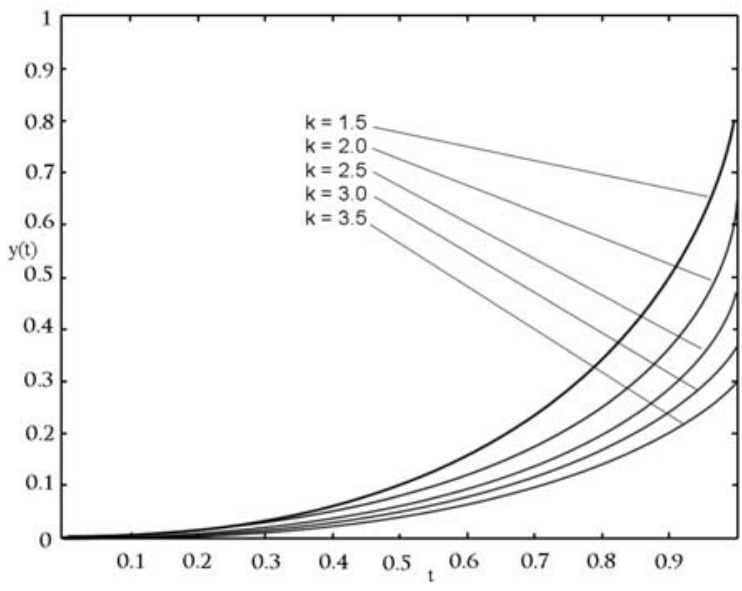

Fig. 1 Pursuit curves for the case when the pursued moves in a straight line

\section{Example 2}

Suppose four mice are located at each of the four corners of a square table, and each mouse runs toward the one to its right. Find the parametric curves that describe the trajectory of the mice. Realize of course, that the velocity of each mouse does not matter; they will still follow the same trajectory.

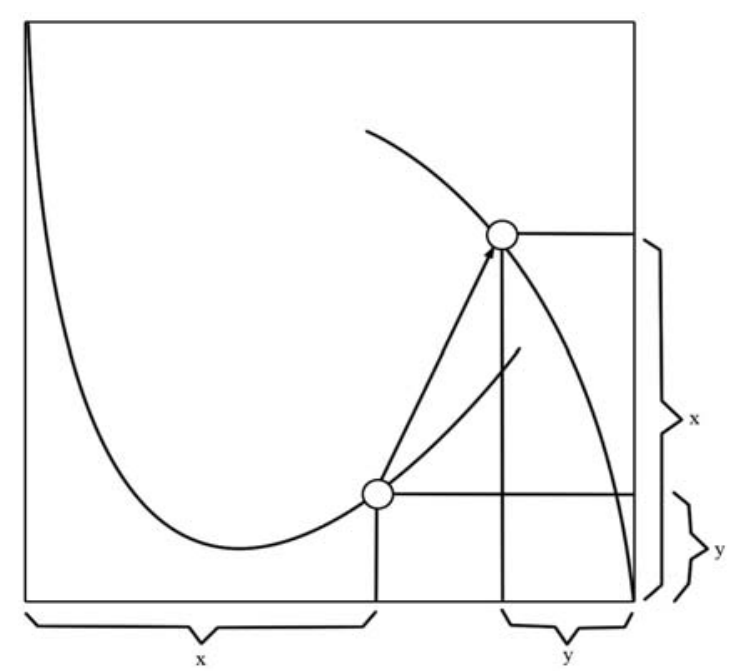

Fig. 2 Actual trajectory of two mice

Solution. We begin with a diagram of the mice on the table. Let the table be of size unity on each side and we concentrate on the bottom left mouse. We can express the slope of its present velocity vector as

$$
\frac{d y}{d x}=\frac{x-y}{1-x-y} .
$$


To introduce the parametric velocities, we can instead write

$$
\frac{\frac{d y}{d t}}{\frac{d x}{d t}}=\frac{x-y}{1-x-y} .
$$

From (2.2) we have

$$
\begin{aligned}
& \frac{d y}{d t}=x-y \\
& \frac{d x}{d t}=1-x-y
\end{aligned}
$$

Laplace transforming this set, and using the initial conditions $y(0)=0$ and $x(0)=0$,

$$
\begin{aligned}
& s Y(s)=X(s)-Y(s) \\
& s X(s)=\frac{1}{s}-X(s)-Y(s) .
\end{aligned}
$$

Solving for $X(s)$ using the first equation, we have $X(s)=(s+$ $+1) Y(s)$. Substituting this into the second, $s(s+1) Y(s)=1 / s-$ $-(s+1) Y(s)-Y(s)$.

Solving for $Y(s)$, and by a similar process for $X(s)$ and inverse transforming we have

$$
\begin{aligned}
& y(t)=\frac{1}{2}-\frac{1}{2} e^{-t} \cos t-\frac{1}{2} e^{-t} \sin t \\
& x(t)=\frac{1}{2}-\frac{1}{2} e^{-t} \cos t+\frac{1}{2} e^{-t} \sin t
\end{aligned}
$$

And we are done. In this process, no calculus has been employed. The hardest part was the algebraic manipulation or looking up the transforms in a table. We may find the others by the same process, but using a different initial condition.

If $y(0)=1$, this would make $y^{\prime}(t) \rightarrow s Y(s)-1$, etc. If a geometric or symmetry argument is used, then each successive trajectory has as its y-component the current x-component, as its x-component one minus the current y-component. To write it all out,

$$
\begin{aligned}
& x_{1}(t)=\frac{1}{2}-\frac{1}{2} e^{-t} \cos t+\frac{1}{2} e^{-t} \sin t \\
& y_{1}(t)=\frac{1}{2}-\frac{1}{2} e^{-t} \cos t-\frac{1}{2} e^{-t} \sin t
\end{aligned}
$$

$x_{2}(t)=\frac{1}{2}+\frac{1}{2} e^{-t} \cos t+\frac{1}{2} e^{-t} \sin t$

$y_{2}(t)=\frac{1}{2}-\frac{1}{2} e^{-t} \cos t+\frac{1}{2} e^{-t} \sin t$

$x_{3}(t)=\frac{1}{2}+\frac{1}{2} e^{-t} \cos t-\frac{1}{2} e^{-t} \sin t$

$y_{3}(t)=\frac{1}{2}+\frac{1}{2} e^{-t} \cos t+\frac{1}{2} e^{-t} \sin t$

$x_{4}(t)=\frac{1}{2}-\frac{1}{2} e^{-t} \cos t-\frac{1}{2} e^{-t} \sin t$

$y_{4}(t)=\frac{1}{2}+\frac{1}{2} e^{-t} \cos t-\frac{1}{2} e^{-t} \sin t$

The plot of the solution is given below.

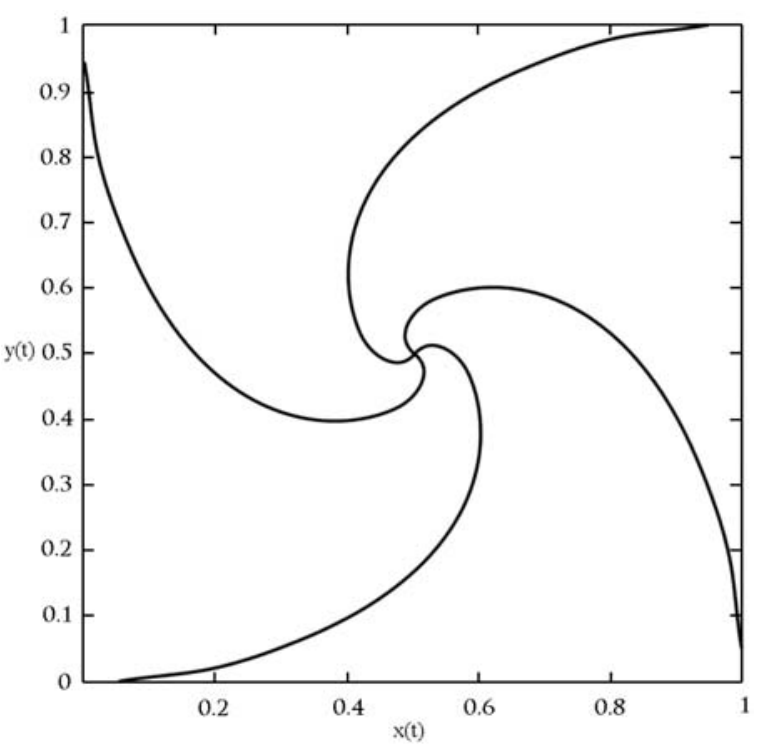

Fig. 3 The curves which describe the trajectory of four mice

\section{Acknowledgement:}

This research has been supported through the project $046 \mathrm{ZU}$ $4 / 201$.

\section{References}

[1] GRAY, A.: Modern Differential Geometry of Curves and Surfaces with Mathematica, $2^{\text {nd }}$ ed., CRC Press, 1998, pp. 66-69.

[2] NAHIN, P. J.: Chases and Escapes: The Mathematics of Pursuit and Evasion. Princeton-New Jersey : Princeton University Press, 2007.

[3] BERNHART, ARTHUR: Curves of General Pursuit, Scripta Mathematica, 24, 1959, pp. 180-206.

[4] <http://mathworld.wolfram.com/topics/ApolloniusPursuitProblem.html. 